\title{
Desempenho Econômico Ambiental dos Municípios de Mato Grosso do Sul: um Estudo dos Indicadores da Contabilidade Verde e Social na Política de Sustentabilidade
}

\section{Environmental Economic Performance of the Municipalities of Mato Grosso do Sul: a Study of Green and Social Accounting Indicators in Sustainability Policy}

\author{
Ayron Vinícius Pinheiro de Assunção*a; Ryan Caldas ${ }^{\mathrm{a}}$; Cristhiano Barbosa ${ }^{\mathrm{a}}$; Alice de Carvalho Assad ${ }^{\mathrm{a}}$
}

${ }^{a}$ Centro Universitário Anhanguera de Campo Grande. MS, Brasil.

*E-mail: ayron.assuncao@anhanguera.com

\begin{abstract}
Resumo
Este artigo tem por finalidade mostrar o desempenho econômico causados pelas principais atividades econômicas do Estado de Mato Grosso do Sul, Brasil. Nesse contexto, os estudos prospectivos irão demonstrar e identificar os indicadores de sustentabilidade de Mato Grosso do Sul para os estudos setoriais e regionais, que subsidiem o aprimoramento de políticas públicas auxiliando na elaboração de Programas Estratégicos de Governo, que levam em consideração as questões do desenvolvimento sustentável ambiental e parâmetros de impacto. Dentro desse enfoque, a possibilidade da construção de indicativos para mensurar quais são os municípios do Estado com maior desempenho econômico sobre o meio ambiente e sobre a sociedade como um todo. Tornando-se relevante na discussão das políticas públicas. Além disso, os diagnósticos gerados pela construção de indicadores sintéticos permitem aos tomadores de decisão cenários prospectivos a respeito da sustentabilidade, subsidiando o aprimoramento, a avaliação e o monitoramento das Políticas Públicas e Programas Governamentais. Para tanto, foi desenvolvida uma abordagem de indicações agregativas para mensuração da sustentabilidade, que avaliou os resultados para os setenta e nove municípios de Mato Grosso do Sul, revelando quais as principais similaridades e pontos de atenção em relação à sustentabilidade. Propondo no geral objetivar, mensurar a construção e estimação de um Índice de Desenvolvimento Sustentável para o Estado.
\end{abstract}

Palavras-chave: Desempenho Econômico. Contabilidade Verde. Desenvolvimento Regional.

\begin{abstract}
This article aims to show the economic performance caused by the main economic activities of the state of Mato Grosso do Sul, Brazil. In this context, prospective studies will demonstrate the identification of Mato Grosso do Sul sustainability indicators for sectoral and regional studies that support the improvement of public policies, aiding in the elaboration of strategic government programs that take into account the issues of sustainable environmental development and impact parameters. Within this approach, it is possible to construct indicators to measure the economic activities with the highest performance on the environment and on society as a whole, becoming relevant in the discussion of public policies. In addition, the diagnoses generated by the synthetic indicators construction allow decision makers to make prospective scenarios regarding sustainability by subsidizing the improvement of the evaluation and monitoring of public policies and government programs. Therefore, an aggregative indicator approach for sustainability measurement was developed, which evaluated the results for the 79 municipalities of Mato Grosso do Sul revealing the main similarities and points of attention related to sustainability, proposing in general, to objectify and measure the construction and estimation of a sustainable development index for the state.
\end{abstract}

Keywords: Economic Performance. Indicators. Environmental Sustainability.

\section{Introdução}

Atualmente, uma das maiores preocupações da humanidade é o desenvolvimento econômico e a sustentabilidade. A construção do entendimento sobre o tema por líderes, em encontros mundiais, destaca a necessidade da geração de novas tecnologias e conhecimentos que visam auxiliar na continuidade da raça humana e das atividades econômicas. Embora não seja recente, os problemas ambientais passaram a ser percebidos, por vários setores da sociedade, se tornando uma preocupação para todos.

Conforme Manzaneres (2005), à medida que cresce o conhecimento científico e técnico sob as causas e efeitos dos danos ambientais, mais a sociedade fica sensibilizada, o que faz aumentar a pressão para as organizações assumirem um novo papel neste contexto. Consequentemente, essa relação impulsiona as empresas a fomentarem o desenvolvimento econômico em conjunto com a preservação do meio ambiente (VELLANI; NAKAO, 2009).

O conceito de desempenho na economia ambiental ainda é novo, o que a torna abstrata, além de apresentar peculiaridades, exigindo assim uma atenção relevante sobre a produção e consumo (entrada-saída) de recursos ambientais, como: água, solo, energia, entre outros. Portanto, é necessário planejar o crescimento econômico com o ambiental com as demais metas estabelecidas, ou seja, um foco no pensamento em longo prazo.

Segundo Assunção (2018) se deve buscar um equilíbrio no desenvolvimento da sociedade e do meio ambiente, de forma 
harmoniosa e que garanta a sustentabilidade entre ambos, pois a preservação do capital natural (meio ambiente) é importante para o crescimento e manutenção sustentável, o que assegura uma melhor condição de vida para as próximas gerações (ASSUNÇÃO, 2018).

Com isso foi gerada uma sociedade mais consciente e preocupada com a questão ambiental sobre poluição do ar, da água e outras questões relacionadas à sustentabilidade, em função de crescente gravidade dos problemas ambientais e a percepção cada vez maior das repercussões desses problemas.

Anteriormente, as formas de se avaliar o desenvolvimento, por meio de indicadores, tinham como base os resultados econômicos. Todavia, por consequência de novas abordagens e pensamentos, a partir de estudos sobre desenvolvimento se rompeu esse conceito que se limita aos aspectos econômicos, abrangendo novas dimensões por perspectivas como sociais, políticas, institucionais e ambientais, relatam Pereira et al. (2011) e Neves e Sauer (2017).

Nesta conjuntura, a exploração de atividades econômicas com impacto ambiental exige mais do que apenas uma fiscalização, mas que incorpore novas tecnologias, processos e programas que buscam nivelar o crescimento econômico e a qualidade ambiental, destaca Neves e Sauer (2017). Dessa forma, Assunção (2018) descreve a contabilidade verde ou ambiental como a incorporação do consumo dos recursos naturais pelas empresas presentes nos registros contábeis.

Para fins de dispositivos legais, foram estabelecidos pela Política Nacional do Meio Ambiente, pelo Decreto $n^{\circ} 4.297 / 2002$, os critérios para o Zoneamento EcológicoEconômico do Brasil (ZEE). Já na esfera Estadual, a presente política é definida pela Lei $n^{\circ} 3.839 / 2009$ que institui o Programa de Gestão Territorial e aprovou a primeira aproximação do ZEE do Estado de Mato Grosso do Sul.

De acordo com Assunção (2018), o Estado de Mato Grosso do Sul tem como normativa do ZEE, que deve ser entendida como um conjunto de ações voltadas para alterações do meio-ambiente, que visam exploração racional dos recursos, que têm como resultado a melhora da qualidade de vida da população e a preservação ambiental do território, realizado pela sociedade em conjunto com o Governo do Estado do Mato Grosso do Sul.

O Balanço Social desenvolvido pelo Instituto Brasileiro de Análises Sociais e Econômicas (IBASE) informa as ações de responsabilidade social desenvolvidas pelas empresas divulgando, de forma transparente, o desempenho de informações contábeis, ambientais, econômicas e sociais das entidades aos seus diferenciados usuários (ASSUNÇÃO, 2018).

Suas características inerentes ao processo de estruturação e organização são essenciais para uma visão mais clara dos processos em que estão envolvidos os custos ambientais, observa Assunção (2018). Sendo necessário escolher indicadores de desempenho ambiental, amenizando as agressões ao meio ambiente e por consequência auxiliando no desempenho econômico.

Nesse novo contexto, os estudos prospectivos para antecipar cumprimento e identificar estratégias para o desenvolvimento de Mato Grosso do Sul são fundamentais para estudos prospectivos setoriais e regionais, que subsidiem o aprimoramento de Políticas Públicas, dando subsídios aos programas estratégicos que levam em consideração as questões da sustentabilidade ambiental.

Dentro desse enfoque, a possibilidade de construção de indicadores para mensurar as responsabilidades do desempenho econômico ambiental fornece para a sociedade, como um todo, informações sobre a situação de suas regiões de um lado e de outro fornece uma ferramenta de tomada de decisão em ações para corrigir desigualdades regionais.

Para tanto, propõe-se como objetivo geral mensurar a estimação de um Índice de Desenvolvimento Sustentável, no âmbito dos municípios no Estado de Mato Grosso do Sul, tendo a construção de um indicador com base na produção e consumo de água.

\section{Material e Métodos}

De acordo com Roldan e Valdés (2002), a metodologia proposta para a seleção do conjunto de indicadores para dar conta de diferentes regiões, possibilitando comparar e gerar um ranking dessas regiões deveria representar a questão do desenvolvimento sustentável. Devendo obedecer aos seguintes critérios: a) a disponibilidade e confiabilidade das fontes de dados; b) a estatística de dado mais atualizada possível e, c) a representação na análise de três sistemas: natural, social e econômico, com sua importância regional.

O método adotado para a coleta de dados, na realização desta pesquisa, ocorre por meio da análise documental, que segundo Casa Nova et al. (2020), é descrita como o levantamento de dados que ainda serão observados para se obter os achados da pesquisa. É uma técnica quantitativa usada para triangulação e/ ou complementar informações obtidas por outros meios ou técnicas, com foco em revelar novos aspectos sobre o tema ora estudado.

Os dados serão coletados da Base de Dados do Estado, visto ser esse um sistema de informações dos municípios do Mato Grosso do Sul, com uma riqueza de dados com classificação por tema ou assunto, que integra a Superintendência de Gestão da Informação - SGI, do Governo do Estado do Mato Grosso do Sul. O sistema é disponibilizado pela Secretária de Estado de Meio Ambiente e Desenvolvimento Econômico (SEMADE).

Já a análise dos dados obtidos ocorrerá por uma abordagem quantitativa, utilizando a técnica de análise estatística descritiva simples, aplicando os dados ora coletados, com a aplicação de uma fórmula baseada na produção e consumo de água por um município, independente do seu fim, ou seja, a produção realizada por empresas públicas ou privadas e 
consumo realizados por todos os habitantes no município, com base na descrição do quadro a seguir:

Quadro 1 - Fórmula de cálculo do indicador de Desempenho do Consumo sobre a Produção de Água

\begin{tabular}{|l|l|}
\hline Fórmula: \\
Sendo: \\
\hline DCP & $\begin{array}{l}\text { Desempenho do Consumo sobre a Produção, em } \\
\text { porcentagem }\end{array}$ \\
\hline VP & Volume Produzido, em metros cúbicos $\left(\mathrm{m}^{3}\right)$ \\
\hline VC & Volume Consumido, em metros cúbicos $\left(\mathrm{m}^{3}\right)$ \\
\hline
\end{tabular}

Para a coleta de dados proposta neste artigo são considerados todos os municípios de Mato Grosso do Sul, com foco na dimensão ambiental propostas pela Comissão de Desenvolvimento Sustentável (CSD) das Nações Unidas e utilizada na metodologia do IDS nacional do IBGE. Sendo os municípios: Água Clara, Alcinópolis, Amambai, Anastácio, Anaurilândia, Angelica, Antônio João, Aparecida do Taboado, Aquidauana, Aral Moreira, Bandeirantes, Bataguassu, Batayporã, Bela Vista, Bodoquena, Bonito, Brasilândia, Caarapó, Camapuã, Campo Grande (Capital), Caracol, Cassilândia, Chapadão do Sul, Corguinho, Coronel Sapucaia, Corumbá, Costa Rica, Coxim, Deodápolis, Dois Irmãos do Buriti, Douradina, Dourados, Eldorado, Fátima do Sul, Figueirão, Gloria de Dourados, Guia Lopes da Laguna, Iguatemi, Inocência, Itaporã, Itaquiraí, Ivinhema, Japorã, Jaraguari, Jardim, Jateí, Juti, Ladário, Laguna Carapã, Maracaju, Miranda, Mundo Novo, Naviraí, Nioaque, Nova Alvorada do Sul, Nova Andradina, Novo Horizonte do Sul, Paraiso das Águas, Paranaíba, Paranhos, Pedro Gomes, Ponta Porã, Porto Murtinho, Ribas do Rio Pardo, Rio Brilhante, Rio Negro, Rio Verde, Rochedo, Santa Rita do Pardo, São Gabriel do Oeste, Selviria, Sete Quedas, Sidrolândia, Sonora, Tacuru, Taquarussu, Terenos, Três Lagoas e Vicentina.

\subsection{Sustentabilidade: aspectos conceituais e históricos}

O conceito de sustentabilidade remete aos princípios básicos como a Democracia ou a Justiça, é um conceito fácil de pronunciar, contudo, complexo de se definir. $\mathrm{Na}$ literatura de economia do meio ambiente, o debate referente às condições de sustentabilidade tende a ser baseado em dois conceitos: sustentabilidade "fraca" e "forte".

De acordo com Pearce e Atkinson (1993) e Pearce e Warford (1995), o teste da sustentabilidade fraca é uma regra intuitiva baseada na hipótese de substituição sem restrições entre ativos produzidos e não produzidos. Uma economia é considerada "não sustentável" se a poupança total fica abaixo da depreciação combinada dos ativos produzidos e não produzidos, os últimos usualmente restritos aos recursos naturais.

Os problemas ambientais podem durar muito tempo, contudo, recentemente, uma parte desses empresários tomaram consciência dos impactos e de suas implicações, seja nos negócios e no meio ambiente. Porém, na literatura os problemas ambientais não foram ignorados pelas diversas escolas do pensamento econômico, um exemplo é a fisiocracia que colocava os recursos naturais (a Terra), em primeiro lugar, entre os fatores de crescimento econômico e a escola clássica considerava os três fatores em conjunto: a Terra, o Capital e o Trabalho.

Foi somente a partir da década de 1970, do século passado, período em que foi realizada grande quantidade de estudos e pesquisas que avançaram a discussão sobre as questões ambientais, na linha econômica neoclássica surgiu duas vertentes que dividiram a Ciência Econômica, sendo essas a Economia Ambiental e Economia dos Recursos Naturais.

A Organização das Nações Unidas, através do relatório Nosso Futuro Comum, definiu Desenvolvimento Sustentável como aquele que busca as necessidades presentes sem comprometer a capacidade das gerações futuras de atender suas próprias necessidades (CMMAD). Então, essa preocupação ganhou corpo, sendo interpretada com um sentido amplo "sustentabilidade", discernindo os meios de produção para buscar um equilíbrio.

Para Ramos (2010), a visão atual de natureza, potencializada pela tecnologia, herdou o projeto de dominação assentado no dualismo homem e natureza, na qual a última é instrumentalizada em benefício do primeiro. Em outras palavras, universalizou-se a postura de transformar o conhecimento da natureza em instrumento de domínio da mesma. A convivência harmoniosa com o ambiente e as demais espécies que habitam no Planeta devem ser respeitadas para que o valor da Educação Ambiental possa ser preservado.

Cordani, Marcovitch e Salati (1997) destacam que problemas de curto prazo acabaram invadindo e lotando as agendas dos líderes cívicos, como o terrorismo e o desemprego têm atraído, significativamente, a atenção dos dirigentes e da sociedade como um todo. Suprimindo outras discussões de grande relevância como o caso das questões ambientais.

No Brasil, as indefinições quanto ao processo de reforma do Estado também têm reduzido a prioridade para as ações do desenvolvimento sustentável. Exemplo desse fato está na inoperância de muitas das instituições dedicadas às questões ambientais e dos seus órgãos de coordenação, incentivados por uma política negacionista e com foco exclusivamente econômico.

Portanto, verificando e respeitando a opinião de cada autor se pode notar que alguns se sujeitaram a expressar e estabelecer o conhecimento de forma clara e objetiva, em função da dificuldade que o assunto pede, sendo assim para garantir o desenvolvimento sustentável é necessário avaliar adequadamente o capital natural. Para não prejudicar as gerações futuras é preciso saber a qual parte do estoque de capital natural foi perdida como resultante da degradação ambiental, quanto é disponível hoje e quanto é ameaçado pela destruição irreversível, no futuro.

O desenvolvimento sustentável é uma ferramenta que 
mostrará informações para agregar os controles, evidenciando os impactos causados ao meio ambiente, conduzindo à elaboração de programas preventivos. Uma prova sobre essa observação é o estudo realizado por Parente, Luca e Romcy (2015), que apontou que as empresas que investem no meio ambiente apresentam um melhor desempenho econômico.

\subsubsection{Indicadores e índices de sustentabilidade}

Não existe um consenso sobre o desenvolvimento sustentável ser um processo evolutivo que se traduz na combinação de três vertentes de desenvolvimento de um país para benefício das gerações presentes e futuras: crescimento da economia, melhoria da qualidade do ambiente e melhoria da sociedade.

Ao se tratar de índices ou indicadores de sustentabilidade, o debate ainda está no iniciando, pois não há, até o presente momento, um índice com um conjunto de indicadores bem definidos para ser avaliar o que é sustentável ou insustentável. Um índice de sustentabilidade deve, inicialmente, se referir aos elementos relativos da sustentabilidade de um sistema (CAMINO; MÜLLER, 1993) e a explicitação de seus objetivos, sua base conceitual e seu público usuário (ROMERO, 2004).

A construção de indicadores de desenvolvimento sustentável no Brasil se integra ao conjunto de esforços internacionais para concretização das ideias e princípios formulados na Conferência das Nações Unidas sobre Meio Ambiente e Desenvolvimento, realizada no Rio de Janeiro em 1992, no que diz respeito à relação entre meio ambiente, sociedade, desenvolvimento e informações para a tomada de decisões.

Para se estabelecer um índice é necessário muito estudo e análise dos dados, inicialmente, primário, e após agregados que se alcançam os subindicadores. Com o agrupamento de vários subindicadores se definem os indicadores para assim determinar subíndices, que por fim organizam os índices, conforme é estabelecido por Shields et al. (2002), adaptado pela figura 1 a seguir:

Figura 1 - Nível de agregação de dados de uma determinada ferramenta de avaliação da sustentabilidade

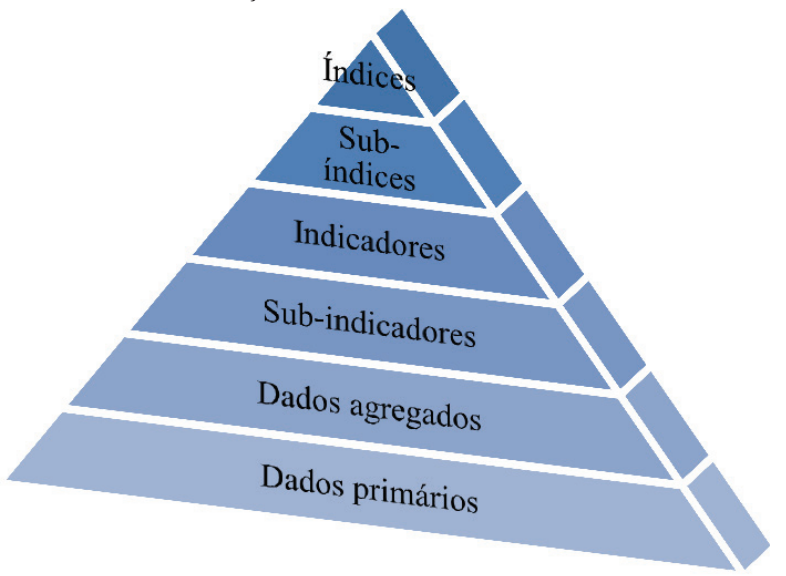

Fonte: adaptado de Shields et al. (2002).
De acordo com a Figura 1, os índices de sustentabilidade implicam na explicação dos mecanismos e lógicas atuantes na área sob análise, quantificação dos fenômenos mais importantes que ocorrem no sistema.

\subsubsection{Construção de indicadores}

Segundo Januzzi (2005), a construção de um sistema de indicadores para uso nas Políticas Públicas acontece em quatro etapas. Inicia-se na definição do objetivo programático (1), sendo em seguida definidas as dimensões ou ações vinculadas ao objetivo programático (2). A partir daí se buscam dados administrativos e estatísticas públicas (3), que após organizadas (4) se tornam indicadores sociais, conforme mostra a Figura 2.

Figura 2 - Construção de sistema de indicadores para ciclo de Políticas Públicas

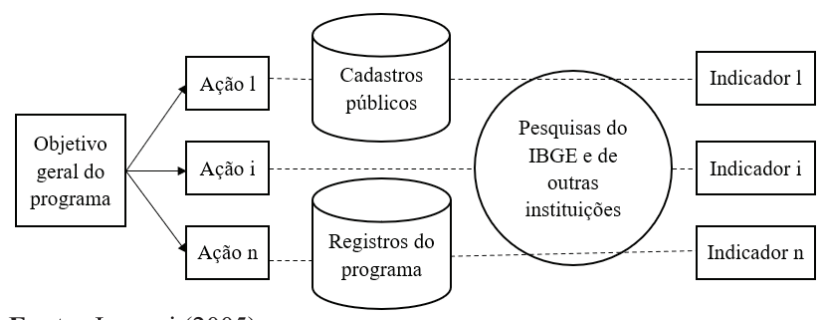

Fonte: Januzzi (2005).

Conforme Januzzi (2005), o indicador deve estar entrelaçado ao planejamento estratégico para que mensure exatamente o que se propõe. Para Cardoso (2005), a esta ligação entre o indicador e o planejamento estratégico ocorre na forma de um ciclo, no qual o planejamento estratégico alimenta a mensuração dos resultados do indicador, que por sua vez utiliza a informação sobre desempenho para alimentar o planejamento estratégico, conforme mostra a Figura 3.

Figura 3 - Ligação entre indicador e planejamento estratégico

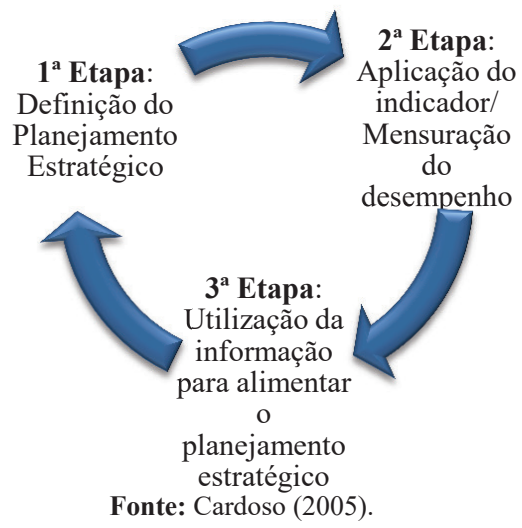

A primeira etapa, a definição de um objetivo programático se faz muito importante para se concretizar a efetividade do indicador, pois a partir da definição do objetivo pragmático o indicador passa a ter uma base conceitual bem definida, aumentando sua eficácia (JANUZZI, 2005).

A partir da definição do conteúdo programático, a segunda etapa é a definição das dimensões que comporão o indicador. Estas dimensões também são chamadas de "grandes temas", 
como foi o caso dos três indicadores trabalhados no Índice de Desenvolvimento Sustentável do Zoneamento EcológicoEconômico de Mato Grosso do Sul. Nesta etapa, é vital que sejam verificadas todas as dimensões que comporão o indicador. Após verificadas, serão então detalhadas aquelas dimensões que se mostrarem essenciais ao indicador e descartadas as que se mostrarem desnecessárias. Esse julgamento ficará a cargo do pesquisador, devendo este buscar conhecimento sobre o assunto para tal realização (JANUZZI, 2005).

A terceira etapa, que Januzzi (2005) define como a busca por dados administrativos e estatísticas públicas, ou seja, a coleta de dados para mensurar um fenômeno. A coleta de dados pode ser realizada por meio de dados primários ou secundários. Os dados primários são aqueles dados inéditos, ou seja, que necessita a realização de coleta. Os dados secundários, por sua vez, são aqueles dados já existentes, que foram coletados e disponibilizados por outro pesquisador ou instituição em base de dados próprios.

A quarta e última etapa se trata da organização dos dados, padronizados quando necessário, utilizando os dados para mensurar o que é desejado. A metodologia de construção de um indicador é apresentada na Figura 4.

Figura 4 - Metodologia de construção de um indicador

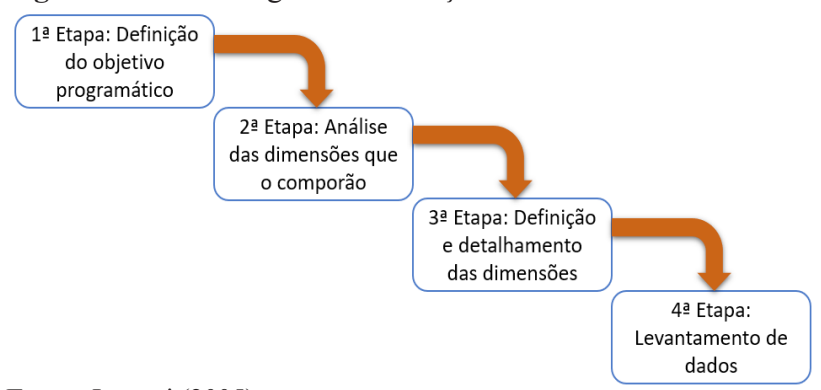

Fonte: Januzzi (2005).

O principal fator negativo dos dados primários em relação aos secundários está no custo de obtenção (financeiro e de tempo) e na dificuldade de obtenção dos dados, uma vez que grande parte das pesquisas necessita alcançar uma população grande. Os dados secundários são mais fáceis de acessar, garantindo economia de tempo e dinheiro. Por outro lado, os dados secundários devem ser utilizados apenas quando o pesquisador tiver certeza da confiabilidade destes dados, dando preferência para dados de instituições de pesquisa como o IBGE ou outras instituições confiáveis.

\section{Resultados e Discussão}

Com base nas informações e nos estudos anteriormente apresentados foi realizada uma correlação da dimensão analítica do indicador Desempenho do Consumo sobre a Produção, para que seja mais claro quais são as dimensões consideradas mais importante. Criando um ranking dos municípios se verifica o desempenho dos setenta e nove municípios do Estado de Mato Grosso do Sul, em ordem alfabética com os resultados no consumo sobre sua produção e com isso a média em relação ao desempenho individual econômico de cada e um geral do Estado (Quadro 2).

Quadro 2 - Base do volume produzido, consumido e o desempenho do consumo sobre produção

\begin{tabular}{|c|c|c|c|c|}
\hline $\mathbf{N}^{\mathbf{o}}$ & Município: & $\begin{array}{c}\text { Volume } \\
\text { Produzido } \\
\text { de Água no } \\
\text { Ano de } 2017 \\
\left(\mathrm{~m}^{3}\right)\end{array}$ & \begin{tabular}{|c|} 
Volume \\
Consumido \\
de Água no \\
Ano de 2017 \\
$\left(\mathrm{~m}^{3}\right)$ \\
\end{tabular} & $\begin{array}{c}\text { Desempenho } \\
\text { do Consumo } \\
\text { sobre } \\
\text { Produção } \\
(\%)\end{array}$ \\
\hline 01 & Água Clara & 886.000 & 646.000 & 0,729 \\
\hline 02 & Alcinópolis & 221.000 & 171.000 & 0,774 \\
\hline 03 & Amambai & 1.717 .000 & 1.227 .000 & 0,715 \\
\hline 04 & Anastácio & 1.464 .000 & 947.000 & 0,647 \\
\hline 05 & Anaurilândia & 403.000 & 301.000 & 0,747 \\
\hline 06 & Angélica & 660.000 & 532.000 & 0,806 \\
\hline 07 & Antônio João & 475.000 & 294.000 & 0,619 \\
\hline 08 & $\begin{array}{l}\text { Aparecida do } \\
\text { Taboado }\end{array}$ & 1.614 .000 & 1.029 .000 & 0,638 \\
\hline 09 & Aquidauana & 2.800 .000 & 1.879 .000 & 0,671 \\
\hline 10 & Aral Moreira & 495.000 & 294.000 & 0,594 \\
\hline 11 & Bandeirantes* & - & - & 0,000 \\
\hline 12 & Bataguassu & 1.362 .000 & 1.090 .000 & 0,800 \\
\hline 13 & Batayporã & 655.000 & 474.000 & 0,724 \\
\hline 14 & Bela Vista* & - & - & 0,000 \\
\hline 15 & Bodoquena & 627.000 & 287.000 & 0,458 \\
\hline 16 & Bonito & 1.686 .000 & 959.000 & 0,569 \\
\hline 17 & Brasilândia & 648.000 & 528.000 & 0,815 \\
\hline 18 & Caarapó & 1.645 .000 & 1.002 .000 & 0,609 \\
\hline 19 & Camapuã & 927.000 & 606.000 & 0,654 \\
\hline 20 & $\begin{array}{l}\text { Campo } \\
\text { Grande } \\
\text { (Capital) } \\
\end{array}$ & 82.522 .080 & 50.902 .631 & 0,617 \\
\hline 21 & Caracol & 257.000 & 166.000 & 0,646 \\
\hline 22 & Cassilândia* & - & - & 0,000 \\
\hline 23 & $\begin{array}{l}\text { Chapadão do } \\
\text { Sul }\end{array}$ & 2.033 .000 & 1.483 .000 & 0,729 \\
\hline 24 & Corguinho* & - & - & 0,000 \\
\hline 25 & $\begin{array}{l}\text { Coronel } \\
\text { Sapucaia } \\
\end{array}$ & 390.000 & 282.000 & 0,723 \\
\hline 26 & Corumbá & 11.663 .000 & 3.966 .000 & 0,340 \\
\hline 27 & Costa Rica* & - & - & 0,000 \\
\hline 28 & Coxim & 2.403 .000 & 1.712 .000 & 0,712 \\
\hline 29 & Deodápolis & 881.000 & 666.000 & 0,756 \\
\hline 30 & $\begin{array}{l}\text { Dois Irmãos } \\
\text { do Buriti }\end{array}$ & 404.000 & 287.000 & 0,710 \\
\hline 31 & Douradina & 267.000 & 206.000 & 0,772 \\
\hline 32 & Dourados & 22.480 .000 & 10.502 .000 & 0,467 \\
\hline 33 & Eldorado & 680.000 & 563.000 & 0,828 \\
\hline 34 & Fátima do Sul & 1.851 .000 & 1.000 .000 & 0,540 \\
\hline 35 & Figueirão & 168.000 & 109.000 & 0,649 \\
\hline 36 & $\begin{array}{l}\text { Glória de } \\
\text { Dourados* }\end{array}$ & - & - & 0,000 \\
\hline 37 & $\begin{array}{l}\text { Guia Lopes da } \\
\text { Laguna }\end{array}$ & 648.000 & 404.000 & 0,623 \\
\hline 38 & Iguatemi & 732.000 & 521.000 & 0,712 \\
\hline 39 & Inocência & 427.000 & 286.000 & 0,670 \\
\hline 40 & Itaporã & 1.200 .000 & 790.000 & 0,658 \\
\hline 41 & Itaquiraí & 665.000 & 527.000 & 0,792 \\
\hline
\end{tabular}




\begin{tabular}{|c|c|c|c|c|}
\hline $\mathbf{N}^{\mathbf{o}}$ & Município: & \begin{tabular}{|c|} 
Volume \\
Produzido \\
de Água no \\
Ano de 2017 \\
$\left(\mathrm{~m}^{3}\right)$ \\
\end{tabular} & \begin{tabular}{|c|}
$\begin{array}{c}\text { Volume } \\
\text { Consumido } \\
\text { de Água no } \\
\text { Ano de } 2017 \\
\left(\mathbf{m}^{3}\right)\end{array}$ \\
\end{tabular} & \begin{tabular}{|c} 
Desempenho \\
do Consumo \\
sobre \\
Produção \\
$(\%)$
\end{tabular} \\
\hline 42 & Ivinhema & 1.614 .000 & 1.296 .000 & 0,803 \\
\hline 43 & Japorã & 102.000 & 82.000 & 0,804 \\
\hline 44 & Jaraguari* & - & - & 0,000 \\
\hline 45 & Jardim & 1.536 .000 & 1.147 .000 & 0,747 \\
\hline 46 & Jateí & 196.000 & 137.000 & 0,699 \\
\hline 47 & Juti & 257.000 & 214.000 & 0,833 \\
\hline 48 & Ladário & 1.581 .000 & 697.000 & 0,441 \\
\hline 49 & $\begin{array}{l}\text { Laguna } \\
\text { Carapã }\end{array}$ & 239.000 & 164.000 & 0,686 \\
\hline 50 & Maracaju & 3.591 .000 & 1.911 .000 & 0,532 \\
\hline 51 & Miranda & 1.027 .000 & 708.000 & 0,689 \\
\hline 52 & Mundo Novo & 1.154 .000 & 908.000 & 0,787 \\
\hline 53 & Naviraí & 3.560 .000 & 2.654 .000 & 0,746 \\
\hline 54 & Nioaque & 540.000 & 374.000 & 0,693 \\
\hline 55 & \begin{tabular}{|l|} 
Nova \\
Alvorada do \\
Sul \\
\end{tabular} & 1.143 .000 & 827.000 & 0,724 \\
\hline 56 & $\begin{array}{l}\text { Nova } \\
\text { Andradina } \\
\end{array}$ & 2.950 .000 & 2.543 .000 & 0,862 \\
\hline 57 & \begin{tabular}{|l|} 
Novo \\
Horizonte do \\
Sul \\
\end{tabular} & 181.000 & 166.000 & 0,917 \\
\hline 58 & \begin{tabular}{|l|}
$\begin{array}{l}\text { Paraíso das } \\
\text { águas* }\end{array}$ \\
\end{tabular} & - & - & 0,000 \\
\hline 59 & Paranaíba & 2.288 .000 & 1.514 .000 & 0,662 \\
\hline 60 & Paranhos & 320.000 & 239.000 & 0,747 \\
\hline 61 & Pedro Gomes & 483.000 & 337.000 & 0,698 \\
\hline 62 & Ponta Porã & 6.187 .000 & 3.320 .000 & 0,537 \\
\hline 63 & $\begin{array}{l}\text { Porto } \\
\text { Murtinho }\end{array}$ & 594.000 & 436.000 & 0,734 \\
\hline 64 & $\begin{array}{l}\text { Ribas do Rio } \\
\text { Pardo }\end{array}$ & 1.114 .000 & 737.000 & 0,662 \\
\hline 65 & Rio Brilhante & 3.115 .000 & 1.668 .000 & 0,535 \\
\hline 66 & Rio Negro & 368.000 & 226.000 & 0,614 \\
\hline 67 & $\begin{array}{l}\text { Rio Verde de } \\
\text { Mato Grosso }\end{array}$ & 1.210 .000 & 876.000 & 0,724 \\
\hline 68 & Rochedo* & - & - & 0,000 \\
\hline 69 & $\begin{array}{l}\text { Santa Rita do } \\
\text { Pardo }\end{array}$ & 259.000 & 199.000 & 0,768 \\
\hline 70 & $\begin{array}{l}\text { São Gabriel } \\
\text { do Oeste* }\end{array}$ & - & - & 0,000 \\
\hline 71 & Selvíria & 405.000 & 276.000 & 0,681 \\
\hline 72 & Sete Quedas & 579.000 & 375.000 & 0,648 \\
\hline 73 & Sidrolândia & 2.461 .000 & 1.456 .000 & 0,592 \\
\hline 74 & Sonora & 949.000 & 742.000 & 0,782 \\
\hline 75 & Tacuru & 225.000 & 187.000 & 0,831 \\
\hline 76 & Taquarussu & 226.000 & 158.000 & 0,699 \\
\hline 77 & Terenos & 1.004 .000 & 506.000 & 0,504 \\
\hline 78 & Três Lagoas & 10.083 .000 & 6.852 .000 & 0,680 \\
\hline 79 & Vicentina & 371.000 & 266.000 & 0,717 \\
\hline 80 & \begin{tabular}{|l} 
Estado do \\
Mato Grosso \\
do Sul
\end{tabular} & 199.868 .080 & 120.836 .631 & 0,605 \\
\hline
\end{tabular}

*Não tem dados publicados

Fonte: dados da pesquisa.

Conforme o Quadro 2 se verifica que o Estado, de forma geral, produz um volume de água muito maior que o seu consumo o que revela estar em um grau de sustentabilidade nesta diretriz, consumindo aproximadamente $60,5 \%$ da produção. Fica claro que ao apurar o município de Campo Grande, por ser a capital, detém dos maiores volumes de produção e consumo de água no Estado, sendo 82.522.080 e $50.902 .631 \mathrm{~m}^{3}$, respectivamente, obtendo um DCP de 0,617, próximo da média do Estado.

Já entre as cidades do interior, os cinco municípios com maior volume de produção são Dourados, Corumbá, Três Lagoas, Ponta Porã e Maracaju que somados produzem cerca de $54.004 .000 \mathrm{~m}^{3}$ de água o que equivale a um quarto de toda a produção do Estado. Agora o top cinco de municípios que mais consomem água é Dourados, Três Lagoas, Corumbá, Ponta Porã e Naviraí, utilizando um total de $27.294 .000 \mathrm{~m}^{3}$ de água, proporcionalmente um quinto do consumo em Mato Grosso do Sul.

Da amostra inicial de setenta e nove municípios, contudo, dez municípios não tiveram os dados publicados o que limitou, parcialmente, a apuração dos dados, de forma mais completa, os municípios desconsiderados nesta análise foram: Bandeirantes, Bela Vista, Cassilândia, Corguinho, Costa Rica, Glória de Dourados, Jaraguari, Paraíso das Águas, Rochedo e São Gabriel do Oeste.

Dos resultados apresentados se verifica que Novo Horizonte do Sul tem o maior Desempenho de Volume por Produção em metros cúbicos no ano de 2017, tendo um DCP de 0,917 , o que revela que a cidade consome praticamente toda a sua produção de água, tornando a menos sustentável sob aspectos de uma estiagem mais rigorosa não possuir recursos hídricos para atender a sua população.

Isso demonstra que pelo indicador ambiental se pode revelar resultados individuais aos municípios, mas que devem ser analisados os resultados parciais do indicador que compõe a dimensão do DCP para traçar estratégias acertadas nas áreas social, econômica, institucional e ambiental. Conforme os resultados apresentados se pode verificar que Dourados tem o maior volume de produção em $\mathrm{m}^{3} \mathrm{em} 2017$. Entretanto, podese verificar que municípios como Bodoquena tem um baixo consumo em $\mathrm{m}^{3}$.

Pelo indicador ambiental DCP se podem mostrar os resultados individuais aos municípios, mas se deve atentar para os resultados parciais dos indicadores que compõem as dimensões para traçar estratégias acertadas nas áreas social, econômica, institucional e ambiental. Com relação aos desempenhos econômicos do consumo no ranking, o Quadro 2 são apresentados os resultados pela fórmula do Volume Produzido de água $\mathrm{em}^{3}$ dividido pelo volume consumido total de água $\mathrm{em}^{3}$.

Analisando o Quadro 2 se pode verificar que Bataguassu possui o menor DCP em relação ao comportamento de suas ações econômicas, dimensionando que apresenta valores no ano de 2017 em 0,800. No entanto, o ranking mostra que a cidade de Novo Horizonte do Sul demonstra ter um forte 
desempenho frugal em suas atividades financeiras, mas em termos da dimensão de consumo por $\mathrm{m}^{3}$ tem desempenho considerado tacanho de $166.000 \mathrm{~m}^{3}$.

Portanto, em análise do indicador sintético ambiental se notam os resultados individuais aos municípios, todavia se devem analisar as repercussões fracionadas dos indicadores que compõem as proporções para traçar métodos pertinentes nas áreas social, econômica, institucional e ambiental. Pelo que foi observado, apenas o indicador relacionado ao DCP foi analisado. Assim sendo, focar nesse indicador.

Com esses resultados abrange o debate e cumpre com o objetivo apresentado. Essa pesquisa vem com a proposta de se abrir a discussão e propor a criação de indicadores e um índice para a Contabilidade Verde e a sustentabilidade dos municípios e empresas, além de auxiliar o Poder Público em criar Políticas para o meio ambiente.

\section{Conclusão}

O esforço do desenvolvimento sustentável no Estado de Mato Grosso do Sul vem buscando atender uma diretriz do Governo Federal da República Federativa do Brasil, passando a atender na divulgação de suas informações. Em razão disso, a contabilidade passou a alinhar seus dados financeiros sociais e ambientais. Isso faz com que a Região sul-mato-grossense tenha seu desempenho econômico de maneira racional e sustentável.

Entretanto, para acompanhar tal desenvolvimento e verificar se isso tem ocorrido, de forma sustentável, o Estado propõe um sistema composto por indicadores ambientais, que integrados formam uma importantíssima ferramenta para o acompanhamento da Política de avanço parcimonioso.

Pode-se afirmar, portanto, que quando se trata de competitividade no Estado de Mato Grosso do Sul se tem Novo Horizonte do Sul, isolado, se mostrando o município com maior média de desempenho módico apresentando valores de 0,917 no ano de 2017, seguido pelo grupo composto por Nova Andradina, Juti e Tacuru, que se destacam a respeito da competitividade, estando todos estes com performance financeira dentro do intervalo 0,862 a 0,831 , sendo os mais competitivos, destacando-se dos demais setenta e nove municípios do Estado, que apresentam desempenho econômico entre 0,340 a 0,540 , se mostrando pouco competitivo em relação a esses cinco primeiros.

Como conclusão se acredita que este artigo tenha criado um indicador de desempenho ambiental confiável e válido, mostrando o nível de eficiência e eficácia para a contabilidade, servindo para assinalar tendências e mostrar a realidade da competitividade no Estado de Mato Grosso do Sul.

Como consequência se espera que o mesmo possa contribuir para a progressão econômica, social e ambiental de Mato Grosso do Sul, podendo servir de ferramenta essencial para o seu aperfeiçoamento, tendo grande valia no acompanhamento do desenvolvimento sustentável regional.

\section{Referências}

ASSUNÇÃO, A.V.P. Indicadores da contabilidade verde $e$ social na política de sustentabilidade no Estado do mato Grosso do Sul, Brasil. Dissertação (Mestrado em Meio Ambiente e Desenvolvimento Regional) - Universidade AnhangueraUniderp, Campo Grande, 2018.

BRASIL. Decreto n. 4.297, de 10 de julho de 2002. Regulamenta o art. $9^{\circ}$, inciso II, da Lei n. 6.938, de 31 de agosto de 1981, estabelecendo critérios para o Zoneamento Ecológico-Econômico do Brasil - ZEE, e dá outras providências. Disponível em: $<\mathrm{http}: / /$ www.planalto.gov.br/ccivil_03/decreto/2002/d4297.htm>. Acesso em: 28 out. 2020.

CAMINO, R.; MÜLLER, S. Sostenibilidad de la agricultura y los recursos naturales: bases para establecer indicadores. San José: IICA/GTZ, 1993.

CARDOSO JÚNIOR, W.F. Inteligência empresarial estratégica: métodos de implantação de Inteligência Competitiva em organizações. Tubarão: Unisul, 2005.

CASA NOVA, S.P.C. et al. Trabalho de Conclusão de Curso. São Paulo: Saraiva, 2020.

CLAUDE, M. Cuentas pendientes: Estado y Evolución de las Cuentas del 1 medio ambiente en América Latina. Ecuador. Fundación Futuro Latinoamericano, 1997.

CMMAD - Comissão Mundial sobre Meio Ambiente e Desenvolvimento. Nosso futuro comum. Rio de Janeiro: Fundação Getúlio Vargas, 1991.

CORDANI, U.G.; MARCOVITCH, J.; SALATI, E. Avaliação das ações brasileiras após a Rio-92. Estud. Avançados, v.11, n.29, p.399-408, 1997.

IBASE - Instituto Brasileiro de Análises Sociais e Econômicas. Disponível em: <http://ibase.br/pt/> Acesso em: 16 set. 2020.

IBGE. Instituto Brasileiro de Geografia e Estatística. Indicadores de Desenvolvimento Sustentável: Brasil 2016. Rio de Janeiro: IBGE, 2016.

JANNUZZI, P.M. Indicadores para diagnóstico, monitoramento e avaliação de programas sociais no Brasil. Rev. Serviço Público, Brasília, v. 56, n. 2, p. 137160, 2005.

MANZANARES, R. A. Stakeholder, entorno y gestión medioambiental de la empresa: la moderacións del entorno sobre la relacións entre la integración de lós stakeholders y lãs estratégias medioambientales. (Tese de Doutorado) - Universidad de Granada, Granada, 2005.

MARTINS, M.F.; CANDIDO, G.A. Índice de Desenvolvimento Sustentável para Municípios (IDSM): metodologia para análise e cálculo do IDSM e classificação dos níveis de sustentabilidade uma aplicação no Estado do Meio Ambiente em América Latina. Quito: Fundación Futuro.

NEVES, T. A.; SAUER, L. Zoneamento Ecológico-Econômico como política pública para o Estado de Mato Grosso do Sul. Interações, Campo Grande, v. 18, n. 3, p. 131-140, 2017.

PARENTE, P.H.N.; LUCA, M.M.M.; ROMCY, T. O. Desempenho ambiental e desempenho econômico de empresas listadas na BM\&F Bovespa. Organizações e Sustentabilidade, v.3, n.2, p.81-115. 2015.

PEARCE, D.W. ATKINSON, G. Capital theory and the mesurement of sustainable development: in: Indicator of weak sustentability. Ecological Economics, v.8, n.2, p.85-103. 1995.

PEARCE, D. W.; WARFORD, J. J. World without end: economics, environment, and sustainable development. Washington: World Bank, 1993. 
PEREIRA, J.R. et al. Gestão social dos territórios da cidadania: o zoneamento ecológico-econômico como instrumento de gestão do território Noroeste de Minas Gerais. Cad. EBAPE.BR, v.9, n.3, p.725-747, 2011.

PFITSCHER, E.D. Contabilidade e responsabilidade social. Florianópolis: UFSC, 2009.

RAMOS, E. C. O processo de constituição das concepções de natureza: uma contribuição para o debate na Educação Ambiental. Rev. Amb. Educ., v.15, p.67-91, 2010.

ROLDÁN, A.B.; SALDÍVAR-VALDÉS, A. Proposal and application of a Sustainable Development Index. Ecological Indicators, , v.2, n.3, p.251-256, 2002.

ROMERO, M. A. B. A Urbanismo Sustentável para a reabilitação de áreas degradadas. Relatório de produtividade de pesquisa 2001-2004, CNPq - UnB/ METRÔ DF, 2004. p.47 -62.

SHIELDS, D.; SOLAR, S.; MARTIN, W. The role of values and objectives in communicating indicators of sustainability. Ecological Indicator, v. 2, n. 1-2, p. 149-160, 2002.

TINOCO, J.E.P. KRAEMER, M.E.P. Contabilidade e Gestão Ambiental. São Paulo: Atlas, 2006.

VELLANI, C.L.; NAKAO, S.H. Investimentos ambientais e redução de custos. Rev. Adm. UNIMEP, v.7, n.2, p.57-75, 2009.

WAQUIL, P. et al. Avaliação de desenvolvimento territorial em quatro territórios rurais no Brasil. Redes, v.15, n.1, p.104-127, 2010. 